\title{
Psychological Distress and Associated Factors in Parents of Children with Cancer
}

\author{
Azizah Othman, Norsarwany Mohamad, Zabidi Azhar Hussin, and Sarah Blunden
}

\begin{abstract}
Introduction: Diagnosis of cancer in children poses a challenging situation for parents to cope with. Method: Seventy-nine $(N=79)$ parents whose children are receiving treatment for cancer in Hospital Universiti Sains Malaysia participated in this cross-sectional descriptive study. They completed a set questionnaire measuring their level of anxiety and stress, knowledge about cancer, and amount of activities they perform with or for the child to enhance the child's coping abilities, in addition to children's psychological problems. Socio-demographic information was obtained. Results: Parents with higher cancer knowledge reported reduced stress $(p<0.01)$ and anxiety $(p<0.05)$. The higher the income $(p<0.05)$ and education $(p<0.01)$, the higher cancer knowledge. Parental stress was negatively correlated with income $(p<0.05)$ and education $(p<0.01)$, indicating that the better educated and higher the salary, less stress symptoms. Highly educated parents engaged in more activities with their children $(p<0.05)$. Parental anxiety was correlated significantly with children's current treatment including chemotherapy procedure $(p<0.01)$, 'In-patient' Vs 'Outpatient' $(p<0.01)$, and children's condition $(p<0.01)$. Parents of hospitalized children who underwent chemotherapy were significantly more anxious than their counterparts. Parents who perceived their children's current condition as 'very good', reported reduced anxiety, compared to those who reported their child's condition as 'ok'. The more psychological problems the children had, the higher parental anxiety $(p<0.05)$ and stress symptoms $(p<0.01)$. Discussion: Some groups of parents reported more psychological difficulties compared to others. Ongoing psychological assessment and intervention may reduce parental stress by increasing coping and reducing children psychological problems.
\end{abstract}

Index Terms - cancer, children, parental knowledge, stress

\section{INTRODUCTION}

Childhood cancer is an increasing and prevalent type of chronic illness worldwide. Malaysian Cancer Registry Report, for instance, indicated that approximately 37 in every 100,000 Malaysian children aged 0 to 15 years are at risk of developing cancer with a slightly higher incidence in males compared to females [1]. With a total population of approximately 27.5 million as in December, 2010 [2], 1,500 children are expected to be diagnosed with cancer every year. Whilst childhood cancer makes up only slightly more than $5 \%$ of the total number of newly reported cancer

cases every year, it is a significant cause of child mortality in the country [3] and the incidence is increasing.

Manuscript received April 3, 2011.

Azizah Othman, Norsarwany Mohamad, and Zabidi Azhar Hussin, Department of Pediatrics, UNIVERSITI SAINS MALAYSIA

Sarah Blunden, Centre for Sleep Research, UNIVERSITY OF SOUTH AUSTRALIA
The adverse effects of childhood cancer on parents' emotional and physical functioning are well documented, especially in the Western countries. A study reviewed 29 articles on the psychological effects of childhood cancer on parents concluded that they experienced significantly more distress as opposed to a normative group of parents [4]. They reported to experience poorer physical and psychosocial quality of life $[5,6]$. The majority of studies suggest that, at some point of time, the parents display a range of emotional challenges especially anxiety and stress symptoms [7] and these two were found to be significantly correlated [8].

There were differences in parental anxiety and quality of life when additional factors are taken into account. For example, anxiety was found to be more prevalent in parents whose children are in active treatment than otherwise [9]. In particular, parents whose children had higher treatment intensity, poorer child health status and shorter time since diagnosis reported poorer quality of life than counterparts [6]. Parents of relapsed children reported higher anxiety levels than parents of surviving and deceased children [10]. This indicates that parents are in need of ongoing psychological support during the course of their child's illness.

It has been established that parents' emotional and behavioral functioning may affect the child's psychological functioning. Highly distressed parents are more likely to have highly distressed children, and vice versa. In pediatric oncology research, similar relationships are evident. Robinson and associates [11] identified significant associations between mothers' and fathers' distress and their reports of their child internalizing symptoms including depression and anxiety. Parents' distress prior to their children undergoing cancer treatment procedures were found to be positively correlated with children's report of pain, whereas parents' concern was negatively associated with children's reported pain and distress [12]. Likewise, there is also an association between parent and child distress outcomes. Specifically, a child's negative functioning can adversely affect his or her parents' psychological adjustment. Maternal psychological problems were more frequently identified among mothers whose children displayed problematic behavior and emotions compared to those children who demonstrated well-functioning behavior. For example, a systematic review that sought to understand care giving experiences in parents of children with cancer, revealed that poorer caregiver psychological well-being were associated with a number of child characteristics including behavior problems, anxiety, depression, and emotional or somatic distress [5]. In short, parent and child could both influence each other's functioning.

In addition to emotional distress, parents' functional 
activities can also be affected. A recent review on the impact of childhood cancer on parents' marital relationship suggests negative changes in parents' relationships, communication, stress, and roles [13]. Multiple sources of stress, changes in the relationship, in addition to other factors like lack of information about the illness or having little knowledge to help the child cope with the current situation $[14,15]$ make the parenting even more challenging.

Previous study has linked knowledge about the illness and psychological distress $[16,17]$. A descriptive study about the level of knowledge in parents of children with cancer indicates that most parents demonstrated a high level of overall pediatric cancer knowledge [18]. The knowledge was significantly associated with educational level and ethnicity, but not with parents' problem-solving ability. In opposition to Orringer et al [17] and Bounduki et al [16], the study indicated that cancer knowledge did not contribute to the variance in psychological adjustment such as depression and mood states.

Therefore, this present study seeks to further examine the relationships between parental knowledge about childhood cancer, their level of anxiety, stress, activities with children, children's problems and selected socio-demographic variables.

\section{METHOD}

\section{A. Participants}

A total of 106 parents whose children were undergoing or who had received cancer treatment at Hospital Universiti Sains Malaysia (HUSM) were approached from October 2006 to June 2007. Parents of terminally-ill children diagnosed during the study period were excluded. Seventy-nine parents (75\%) agreed to participate - all were Muslim, Malay and married (100\%). Sixty percent were mothers and $58 \%$ claimed receiving adequate family support. The majority of the participants had obtained at least 12 years of education (64\%), earned between RM501.00 to RM $3000.00(58 \%)$ and were housewives $(48 \%)$. As for the children, $67 \%$ (Mean age 8.03 ) were males. The majority of them were diagnosed with leukemia $(66 \%)$ within a period of 7 - 36 months $($ Mean $=39.6$ months $)(52 \%)$. During the data collection, $54 \%$ were hospitalized for chemotherapy.

\section{B. Measures}

1) The Cancer Knowledge (CK) questionnaire was constructed based on questionnaires developed in previous studies [18, 19]. A pediatric oncologist examined the items' content validity that resulted in the elimination of one item (i.e. number of hospitals that provide pediatric oncology treatment in Malaysia). The final version of $\mathrm{CK}$ consisted of 11 questions about childhood cancer and its management such as: There are .... types of tumors and Chemotherapy is a treatment that uses ....... Parents completed sentences by choosing 1 of 3 answer choices, 1 point for each correct answer. Higher scores indicated greater cancer knowledge.

2) State Anxiety ( $S A)$ is a subscale of a reliable, widely-used instrument, The State-Trait Anxiety Inventory (STAI)
[20] which consists of 20 statements measuring respondent's present feelings ranging from 1 (not at all) to 4 (very much so). Higher score indicates greater anxiety levels (Range: 20-80). Validation of STAI on a Malaysian sample revealed a high internal consistency $($ Cronbach alpha $=0.86)$ [21]. Coefficient alpha in our sample was 0.93 .

3) Strain Questionnaires ( $S Q$ ) is a 48-item test measuring physical, behavioural, and cognitive symptoms of stress [22] on a scale of 1 (not at all) to 5 (everyday) whereby higher scores that indicates greater stress. Coefficient alpha in the present sample was 0.94 .

4) Parents' Activities with Children (PA) is a list of 29 behavioral activities that parents may perform with or for the child to enhance the child's coping abilities. It was developed from an extensive review on parents' involvement in caring for sick children with reference to Melnyk's Index of Parent Participation at Hospital [23]. Behaviours proven by research to be helpful to the child's coping such as cuddling, distracting and coaching deep breathing $[24,25]$ were listed. To strengthen content validity, the original list of 32 items was examined by 6 specialists including clinical psychologists, paediatricians and paediatric nurses, who suggested modifications. Three items were removed and some statements reworded to facilitate reader's comprehension. One point was given to 'Yes' response and 0 point for other responses (i.e. 'No, Don't Want to Perform, or No Opportunity to Perform'). 'Yes" answers were summed, the highest possible being 29, indicating more activities. Face validity of the PA list was established in an earlier pilot study with 5 parents of children with cancer. Coefficient alpha in the current sample was 0.87 .

5) Strength and Difficulties Questionnaires (SDQ) mainly assesses children's emotional and behavioural problems [26]. Parents responded to items like my child seems worried, on a Likert 3-point scale where $0=$ Not True; 1 = Somewhat True; and $2=$ Certainly True. A preliminary BM version of SDQ as provided by the author [27] was revised by a qualified translator, who also translated the SDQ for this study. The Cronbach alpha for 20 negative items ranged from $0.51-0.66$.

\section{Procedure}

Participants were recruited from oncology clinics, wards and through telephone calls. After informed consent, participants completed the assessment measures with the assistance of the researchers. Ethical approval was granted by Research and Ethics Committees of University of South Australia (UniSA) and HUSM - a government- hospital providing specialized services to Malaysians residing on the north-east coast.

\section{Analyses}

The relationships between five variables (i.e. CK, SA, SQ, PA and SDQ) were investigated using Pearson Product Moment correlations. Supplementary correlation analyses were conducted between those variables and 
socio-demographic characteristics. Finally, the factors predicting stress symptoms among the parents of children with cancer were specifically examined, using a binary logistic regression analysis. Significance was set at $\mathrm{p}<0.05$.

\section{RESULTS}

The range of correlations among the major variables was 0.24 to 0.51 . Parents with more knowledge in childhood cancer indicated reduced scores on SQ $(r=-0.24)$ and SA $(r$ $=-0.36)$. SQ were found to be positively correlated with both SA $(r=0.36)$, and SDQ $(r=0.51)$ indicating that the more psychological problems the children had, the higher parental anxiety and stress symptoms were reported. Parents' activities with the children's scores were not significantly correlated with any other variables. Results are presented in Table I.

TABLE I: CORRELATION MATRIX BETWEEN MEASURES

\begin{tabular}{|l|l|l|l|l|l|l|}
\hline & $n$ & \multicolumn{1}{c}{$C K$} & \multicolumn{1}{|c|}{$S A$} & \multicolumn{1}{c}{$S Q$} & $P A$ & $S D Q$ \\
\hline$C K$ & 73 & - & $-0.24^{*}$ & $-0.36^{* *}$ & 0.20 & $-0.19^{*}$ \\
\hline$S A$ & 76 & - & - & $0.36^{* *}$ & 0.10 & $0.26^{*}$ \\
\hline$S Q$ & 64 & - & - & - & 0.13 & $0.51^{* *}$ \\
\hline$P A$ & 78 & - & - & - & - & 0.19 \\
\hline$S D Q$ & 77 & - & - & - & - & - \\
\hline$S D Q$ & 77 & - & - & - & - & - \\
\hline
\end{tabular}

$\mathrm{CK}=$ Cancer Knowledge; $\mathrm{SA}=$ State Anxiety; $\mathrm{SQ}=$ Strain Questionnaires; SDQ = Strength Difficulties Questionnaires; $\mathrm{PA}^{\wedge}$ Parents’ Activities with Children (using Spearman non-parametric statistics because of non-normal distribution); ${ }^{* *} \mathrm{p}<.01$ (2-tailed); ${ }^{*} \mathrm{p}<.05$ (2-tailed)

The relationships between the five major variables with participants' socio-demographic characteristics were examined. Altogether, there were 60 correlations made on a total of 79 participants. For two continuous variables, Pearson Product Moment Correlation was utilized and for one continuous variable with one ordinal variable, Spearman Rho Correlation was used. When a continuous variable was correlated with a dichotomous variable, we used Point Biserial Pearson Correlation method of analysis. The results are presented in Table II.

TABLE II: CORRELATION BETWEEN MEASURES AND PARTICIPANTS' CHARACTERISTICS

\begin{tabular}{|l|l|l|l|l|l|}
\hline \multicolumn{1}{|c|}{ Variables } & $C K$ & $S A$ & $S Q$ & $P A$ & $S D Q$ \\
\hline Gender $^{+}$ & -0.20 & -0.15 & -0.05 & -0.05 & 0.22 \\
\hline Income $^{\wedge}$ & $0.28^{*}$ & -0.12 & -0.28 & -0.07 & -0.05 \\
\hline Education $^{\wedge}$ & $0.32^{*}$ & 0.03 & $-0.30^{*}$ & $0.26^{*}$ & 0.01 \\
\hline Distance & 0.05 & 0.02 & 0.06 & 0.02 & 0.14 \\
\hline \# Children & -0.14 & -0.28 & 0.01 & -0.04 & -0.01 \\
\hline Support + & 0.22 & 0.21 & 0.03 & 0.02 & 0.03 \\
\hline
\end{tabular}

$\mathrm{CK}=$ Cancer Knowledge; SA = State Anxiety; $\mathrm{SQ}=$ Strain Questionnaires; SDQ = Strength and Difficulties Questionnaires PA $=$ Parents’ Activities with Children; ${ }^{\wedge}$ Spearman Rho Correlation (For Ordinal Data); + Point Biserial Pearson Correlation; ${ }^{* *} \mathrm{p}<.01$ (2-tailed); ${ }^{*} \mathrm{p}<.05$ (2-tailed)

There were positive significant correlations between the $\mathrm{CK}$ and participants' income $(\mathrm{r}=0.28)$ and level of education $(\mathrm{r}=0.32)$, suggesting the higher the income and education level, the higher scores they obtain in CK, and vice versa. By contrast, SQ was found to be negatively correlated with both income $(r=0.28)$ and education $(r=0.30)$, indicating that the better educated and higher the salary, fewer stress symptoms were reported. Parents with higher education engaged in more coping-activities with their children $(r=0.26)$.

The five major variables were then correlated with children's socio-demographic characteristics (see Table III). There were moderate correlations between SA and children's current treatment such as like 'On' Vs 'Off' Chemotherapy $(\mathrm{r}=-0.35)$ 'In-patient' Vs 'Outpatient' $(\mathrm{r}=-0.33)$, and children's condition as perceived by their parents $(r=-0.41)$. Additional comparison analyses using independent t-tests were run on these significantly correlated variables, and revealed that parents of children who were in hospital and undergoing chemotherapy treatment were significantly more anxious than their counterparts. In addition, parents who perceived their children's current condition as 'very good', reported less anxiety, compared to those who reported their child's condition as 'ok'.

TABLE III: CORRELATION BETWEEN MEASURES AND CHILDREN'S

\begin{tabular}{|l|l|l|l|l|l|}
\hline & $C K$ & $S A$ & $S Q$ & $P A$ & $S D Q$ \\
\hline Gender + & 0.01 & -0.11 & -0.17 & -0.08 & -0.05 \\
\hline Age & -0.15 & -0.21 & -0.12 & $-0.29^{*}$ & -0.01 \\
\hline Duration & -0.18 & -0.11 & -0.16 & $-0.28^{*}$ & -0.07 \\
\hline On-chemo+ & 0.16 & $0.35^{* *}$ & -0.03 & $0.27^{*}$ & -0.04 \\
\hline In-patient & -0.14 & $-0.33^{* *}$ & 0.05 & -0.21 & 0.08 \\
\hline Condition+ & 0.16 & $-0.41^{* *}$ & 0.05 & -0.03 & -0.20 \\
\hline
\end{tabular}

$\mathrm{CK}=$ Cancer Knowledge; SA = State Anxiety; SQ $=$ Strain Questionnaires; SDQ $=$ Strength Difficulties Questionnaires, PA $=$ Parents' Activities with Children; ^ Spearman Rho Correlation (For Ordinal Data); + Point Biserial Pearson Correlation; $* * \mathrm{p}<.01$ (2-tailed); ${ }^{*} \mathrm{p}<.05$ (2-tailed)

Additional analyses were conducted to examine factors predicting high levels of stress among the participants (see Table IV).

TABLE VI: LOGISTIC REGRESSION RESULTS IN PREDICTING LIKELIHOOD OF REPORTING HIGH STRESS

\begin{tabular}{|c|c|c|c|c|c|c|c|c|}
\hline $\begin{array}{c}\text { Variabl } \\
e\end{array}$ & $B$ & S.E. & $\begin{array}{c}\text { Wal } \\
d\end{array}$ & $d f$ & $p$ & $\begin{array}{c}\text { Odd } \\
s \\
\text { ratio }\end{array}$ & \multicolumn{2}{|c|}{$\begin{array}{c}C I \\
\text { Lower-Highe } \\
r\end{array}$} \\
\hline$S D Q$ & 0.43 & 0.16 & 7.39 & 1 & 0.007 & 1.54 & 1.13 & $\begin{array}{c}2.1 \\
0\end{array}$ \\
\hline$S A$ & 0.09 & 0.04 & 6.34 & 1 & 0.012 & 1.09 & 1.02 & $\begin{array}{l}1.1 \\
7\end{array}$ \\
\hline$C K$ & $\begin{array}{l}-0.3 \\
7 \\
\end{array}$ & 0.21 & 3.16 & 1 & 0.076 & 0.69 & 0.45 & $\begin{array}{l}1.0 \\
4\end{array}$ \\
\hline Income & $\begin{array}{l}-1.0 \\
6 \\
\end{array}$ & 0.58 & 3.30 & 1 & 0.069 & 0.35 & 0.11 & $\begin{array}{l}1.0 \\
8\end{array}$ \\
\hline Education & $\begin{array}{l}-0.6 \\
2\end{array}$ & 0.53 & 1.40 & 1 & 0.237 & 0.53 & 0.19 & $\begin{array}{l}1.5 \\
0\end{array}$ \\
\hline Constant & $\begin{array}{l}-2.3 \\
9\end{array}$ & $\begin{array}{l}-2.4 \\
5\end{array}$ & 0.95 & 1 & 0.33 & 0.09 & & \\
\hline
\end{tabular}

(Cancer Knowledge), Income, and Education Level.

The dependent variable measuring the stress level was SQ. For the purpose of this analysis, we assigned the SQ as equal to 0 if the parents scored $\leq 85$ (Low stress group) and 1 for those scored $\geq 86$ (High stress group). The binary logistic regression model was performed to estimate factors that influence the high stress group. The model consists of five independent variables, which had been identified to be significantly correlated with SQ namely SDQ, CK, SA, Income and Education Levels. The results from the model indicate that parents reported high stress according to bidirectional relationships of children's and parents' psychological functioning, as seen in previous literature. The coefficient on the SDQ variable is positive and statistically 
significant at the $\mathrm{p}<0.01$ (Wald statistics $=7.39$ ). Parents whose children had behavioral and emotional problems were $1 \frac{1}{2}$ times more likely to report high stress [Odds ratio $=1.54$, $95 \%$ CI $=1.13-2.10)$. Similarly, parents who had high anxiety were marginally more likely to report high stress [Odds ratio $=1.02,95 \% \mathrm{CI}=1.02-1.17$ ] as indicated by the positive and significant SA coefficient, statistically significant at $\mathrm{p}<.05$ (Wald statistics $=6.34$ ). The overall model is significant at $\mathrm{p}<.001\left[\chi^{2}(4, \mathrm{~N}=54)=36.5\right]$, indicating that the model was able to distinguish between high and low stress groups. This five-factor model as a whole explained $65 \%$ (Nagelkerke $\mathrm{R}^{2}$ ) of the variance in stress status. Finally, the model is able to correctly classify $87 \%$ of the cases in either high and low stress group, and thus is acceptable for use in predicting factors related to high level of stress.

In summary, the study examines the correlation of major outcome variables with each other and with socio-demographic variables. The findings suggest some significant inter-relationships. For instance, higher levels of education and income were closely linked to higher scores in knowledge. Higher or more knowledge is also associated with less stress and anxiety. The level of anxiety, in particular, was partly dependent on the children's condition during the time of assessment. For example, parents whose children were not hospitalized and not undergoing chemotherapy were significantly less anxious than their counterparts. Higher anxiety, on the other hand, was correlated with higher children's problems and stress. Finally, a high level of stress was predicted mostly by children's problems. As such, parents who reported their children as having psychological problems were 1.5 times more likely to be classified in the high stress group.

\section{Discussion}

This study, the first of its kind in Malaysia supported the hypotheses of significant negative association between cancer knowledge, anxiety and stress symptoms in parents of children with cancer. The higher the participants' knowledge acquisition scores, the less anxiety and symptoms of stress were reported. These findings are consistent with previous studies indicating an inverse association between psychological distress and illness-related knowledge [16, 17]. It is found that amongst key problems faced by parents whose children are affected by cancer include uncertainty, which is associated with anxiety and post-traumatic stress symptoms [28]. Parents often become uncertain about the controllability of cancer and the complexity of information related to illness and treatments. Thus, providing individuals with adequate information has been demonstrated to reduce uncertainty and to facilitate improved adjustment and coping [29]. Greater knowledge may serve to decrease parental anxiety and distress by helping them to regain a sense of control over circumstances surrounding their children's illness. Findings from a qualitative study on the effect of childhood cancer in Hong Kong Chinese families suggested that the fear of disease was often related to lack of knowledge rather than the disease itself [30]. This study claimed that knowing more about the disease did not necessarily result in increased fear, but improved the parents' psychological well-being. Therefore, in the present study, higher levels of knowledge may have increased parental sense of control over the situation, and thus decreased their state of anxiety and symptoms of stress. Interestingly, the results showed that the greater the knowledge, the more the parents were involved with their child's activities. However, the correlation did not reach significance.

The results in our study showed that better educated individuals scored higher in cancer knowledge as participants with a higher level of education scored higher in CK compared to participants with secondary and primary educational levels. CK was also found to be positively and significantly correlated with participants' socio-economic status as measured by monthly household income. These results are interrelated because those who are better educated are more likely to have higher paid employment. This finding is consistent with previous studies which have also found the level of knowledge about illness tend to be lower among the less educated individuals $[18,31]$.

With regard to anxiety, participants' levels of anxiety correlated significantly with children's current treatments and medical conditions, as perceived by their parents. Group comparison analyses confirmed that parents whose children were in the hospital and receiving chemotherapy were significantly more anxious compared to their counterparts. These findings are consistent with previous studies that indicate that having a hospitalized child at the time of assessment [32] or a child having active treatment such as chemotherapy [33] is associated with more psychological difficulties in the parents, including anxiety. It is no surprise that there was a strong relationship between these factors. Parents whose children are in active treatment, such as chemotherapy, have to face many challenging and anxiety-provoking situations. Parents must deal with their own fear about the uncertainty of the illness and to witness the child enduring the bio-medical effects of the treatment, including pain, aversive procedures (e.g., injections), complex medical regimens, as well as the side-effects of therapy, such as nausea, vomiting, and changes in their child's physical appearance [34]. Furthermore, parents must learn to help the child cope with feelings of fear, anger, or social isolation that are often associated with lengthy hospitalization as required in the treatment of chemotherapy. At the same time, they must maintain their roles as parents to the other children at home. As all their time and attention are focused on the sick child at the hospital, these situations are difficult to manage.

Moreover, the results indicated that parental perception of the children's current condition affects the state of anxiety. Parents, who perceived that their children's condition during the assessment was very good, reported significantly lower levels of anxiety than those who perceive their children's condition as 'ok'. It is possible that this may be related to an optimism/pessimism temperament factor that was not measured in this study.

Further analyses were conducted to uncover significant factors that were related to parental stress. A correlation analysis between parental stress symptoms and all possible independent variables revealed that stress symptoms were 
significantly correlated, and thus may be affected by or from multiple factors including state of anxiety, cancer knowledge, income, education levels, and children's behavioral problems. In particular, high levels of stress on parents in this study were significantly predicted by SA and SDQ, the latter being a main predictor of high parental stress. This study showed that parents who had children with behavioral and emotional problems were 1.5 times more likely to belong in the high stress group. The association between behavioral problems in chronically ill children and parental stress has been established [35] including in Malaysian parents [36]. Indeed, some studies suggest that children's behavioral problems may be a prominent source of stress for the parents, compared to the child's disability [35, 37]. This present finding provides additional support to Klassen [5] which identified child characteristics as one of the most important variable in their care giving process and caregiver burden model.

In summary, the results have shown that the stress symptoms were found to be predicted largely by children's behavioral problems. Higher levels of knowledge were seen in parents with higher education levels and financial status. Parents with a higher level of cancer knowledge also reported less anxiety and stress. There were groups of individuals who were more vulnerable and reported more psychological difficulties compared to others (i.e. had lower levels of education, income and more children at home). This group of people reported more psychological distress, such as stress and anxiety. In addition, parents whose children were hospitalized, receiving chemotherapy and were perceived as being only in reasonably good condition were more anxious than those whose children were not in active treatment and perceived as in a very good medical condition. These parents may be at higher risk of developing psychological problems and they could be identified earlier through psychological screening. The Psychosocial Assessment Tool (PAT) which screens families of children with cancer for potential risks for elevated distress during treatment is an example of available tool [38]. The findings of this study could be used to develop a predictive model of which parents who would most benefit from psychological support and intervention so that those in most need could be targeted. Intensive psychological intervention could then be offered to the most distress subgroups. This could minimize cost and resources in psychological services which are fairly limited especially in Malaysian government hospitals.

\section{LIMITATIONS}

Self-report measure as the only method of assessment used in this study could limit the findings. Whilst it is preferable to gather information from multiple resources, the self-report measure was selected as a logistically suitable method for the pilot community-based study, such as the present one. Whilst the participants represent the local Kelantanese population that consists of $94.2 \%$ Malays and 95.0\% Muslims [39], the group may not entirely represent the Malaysian population overall which consists of Malay, Chinese and Indian ethnic divisions and different religious beliefs. Generalizing the findings to broader ethnic backgrounds or religious groups may be problematic due to a relatively small sample of single race and religion. Therefore, these findings should be considered within that context. Despite these methodological limitations, the importance of this study as the first of its kind in a pediatric oncology setting at a Malaysian government hospital should not be underestimated.

\section{ACKNOWLEDGMENT}

Part of this research is supported by Universiti Sains Malaysia.

\section{REFERENCES}

[1] G. C. C. Lim, H. Yahya, and T. O. Lim, "The First Report of the National Cancer Register Cancer Incidence in Malaysia 2002," National Cancer Registry Ministry of Health Malaysia, Kuala Lumpur July 2003.

[2] Department of Statistics Malaysia, "Preliminary count report of census 2010 - Total Population." retrieved from http://www.statistics.gov.my available online on March 2011.

[3] Ministry of Health Malaysia, "Speech by YB Datuk Seri Dr Chua Soi Lek, Minister of Health Malaysia, ROLF All-Star Charity Dinner, Kuala Lumpur Pavillion ", 2007.

[4] A. L. H. Pai, D. Drotar, K. Zebracki, M. Moore, and E. Youngstrom, "A meta-analysis of the effects of psychological interventions in pediatric oncology on outcomes of psychological distress and adjustment," Journal of Pediatric Psychology, vol. 31, pp. 978-988, 2006.

[5] A. Klassen, P. Raina, S. Reineking, D. Dix, S. Pritchard, and M. O’Donnell, "Developing a literature base to understand the caregiving experience of parents of children with cancer: a systematic review of factors related to parental health and well-being," Supportive Care in Cancer, vol. 15, pp. 807-818, 2007.

[6] A. F. Klassen, R. Klaassen, D. Dix, S. Pritchard, R. Yanofsky, M. O'Donnell, A. Scott, and L. Sung, "Impact of Caring for a Child With Cancer on Parents' Health-Related Quality of Life," Journal of Clinical Oncology, vol. 26, pp. 5884-5889, 2008.

[7] R. Ow, "Burden of care and childhood cancer: Experiences of parents in an Asian context," Health \& Social Work, vol. 28, pp. 232-240, 08 2003.

[8] M. Barrera, N. M. D'Agostino, J. Gibson, T. Gilbert, R. Weksberg, and D. Malkin, "Predictors and mediators of psychological adjustment in mothers of children newly diagnosed with cancer," Psychooncology, vol. 13, pp. 630-641, 2004.

[9] L. S. Larson, D. A. Wittrock, and A. K. Sandgren, "When a child is diagnosed with cancer. I. Sex differences in parental adjustment," Journal of Psychosocial Oncology, vol. 12, pp. 123-142, 1994.

[10] B. J. Wijnberg-Williams, W. A. Kamps, E. C. Klip, and J. E. H. M. Hoekstra-Weebers, "Psychological adjustment of parents of pediatric cancer patients revisited: Five years later," Psycho-oncology, vol. 15, pp. 1-8, 2006.

[11] K. E. Robinson, C. A. Gerhardt, K. Vannatta, and R. B. Noll, "Parent and Family Factors Associated with Child Adjustment to Pediatric Cancer," Journal of Pediatric Psychology, vol. 32, pp. 400-410, 2007.

[12] L. A. Penner, R. J. W. Cline, T. L. Albrecht, F. W. K. Harper, A. M. Peterson, J. M. Taub, and J. C. Ruckdeschel, "Parents' Empathic Responses and Pain and Distress in Pediatric Patients," Basic Appl Soc Psych., vol. 30, pp. 102-113, 2008.

[13] F. M. Da Silva, E. Jacob, and L. C. Nascimento, "CLINICAL SCHOLARSHIP: Impact of Childhood Cancer on Parents' Relationships: An Integrative Review," Journal of Nursing Scholarship, vol. 42 , pp. 250-261, 2010.

[14] I. Coyne, "Parental participation in care: a critical review of the literature " Journal of Advanced Nursing vol. 21, pp. 716-722, 1995.

[15] K. Blower and E. Morgan, "Great expectations? Parental participation in care " Journal of Child Health Care, vol. 4, pp. 60-65, 2000

[16] G. Boundouki, G. Humphris, and A. Field, "Knowledge of oral cancer, distress and screening intentions: longer term effects of a patient information leaflet.," Patient Educ Couns., vol. 53, pp. 71-77, 2004.

[17] J. S. Orringer, A. M. Fendrick, P. C. Trask, C. K. Bichakjian, J. L. Schwartz, T. S. Wang, D. J. Karimipour, and T. M. Johnson, "The effects of a professionally produced videotape on education and anxiety/distress levels for patients with newly diagnosed melanoma: A randomized, prospective clinical trial," Journal of the American Academy of Dermatology, vol. 53, pp. 224-229, 2005. 
[18] V. Shabanzadeh, "Cancer Knowledge, Problem Solving Ability, and Psychological Adjustment in Mothers of Newly Diagnosed Pediatric Cancer Patients," in California School of Professional Psychology. vol. Doctor of Philosophy in Psychology Los Angeles: California School of Professional Psychology, 2001.

[19] [E. R. Katz, O. J. Sahler, M. J. Dolgin, J. W. Varni, R. Mulhern, D. R. Copeland, R. B. Noll, and K. Roghmann, "Cancer Knowledge Inventory," Unpublished Inventory 1997.

[20] C. Spielberger, Manual for the State-Trait Anxiety Inventory. Palo Alto CA: Consulting Psychologists Press, 1983.

[21] K. F. Quek, W. Y. Low, A. H. Razack, C. S. Loh, and C. B. Chua, "Reliability and validity of the Spielberger State-Trait Anxiety Inventory (STAI) among urological patients: A Malaysian study," Medical Journal of Malaysia, vol. 59, pp. 258-267, 2004.

[22] R. C. Lefebvre and S. L. Sandford, "The Strain Questionnaire," in Innovations in Clinical Practice: A Source Book vol. 5, P. A. Keller and L. G. Ritt, Eds. Florida: Professional Resource Exchange, Inc, 1986.

[23] B. M. Melnyk, "Coping with unplanned childhood hospitalization: Effects of informational interventions on mothers and children," Nursing Research, vol. 43, pp. 50-55, 1994.

[24] Y. Pongjaturawit and R. Harrigan, "Parent participation in the care of hospitalized child in Thai and Western Cultures," Issues in Comprehensive Pediatric Nursing vol. 26, pp. 183-199, 2003.

[25] Y. Vance and C. Eiser, "Caring for a child with cancer - A systematic review," Pediatric Blood \& Cancer, vol. 42, pp. 249-253, 2004.

[26] R. Goodman, "Psychometric Properties of the Strengths and Difficulties Questionnaire," Journal of the American Academy of Child \& Adolescent Psychiatry, vol. 40, pp. 1337-1345, 2001.

[27] C. Mortimore, "Information for researchers and professionals about the Strengths and Difficulties Questionnaires," Child and Adolescent Mental Health, vol. 12, pp. 98-98, 2007.

[28] S. Santacroce, "Uncertainty, Anxiety, and Symptoms of Posttraumatic Stress in Parents of Children Recently Diagnosed with Cancer," Journal of Pediatric Oncology Nursing, vol. 19, pp. 104-111, 2002.

[29] C. Hardwick and N. Lawson, "The information and learning needs of the caregiving family of the adult patient with cancer," European Journal of Cancer Care, vol. 4, pp. 118-121, 1995.
[30] L. K. Yin and S. Twinn, "The effect of childhood cancer on Hong Kong Chinese families at different stages of the disease," Cancer Nursing, vol. 27, pp. 17-24, 2004

[31] B. E. Meyerrowitz, J. Richardson, S. Hudson, and B. Leedham, "Ethnicity and cancer ourcomes: Behavioral and Psychosocial Considerations," Psychological Bulletin, vol. 123, pp. 47-70, 1998.

[32] R. K. Mulhern, D. L. Fairclough, B. Smith, and S. M. Douglas, "Maternal depression, assessment methods, and physical symptoms affect estimates of depressive symptomatology among children with cancer," Journal of Pediatric Psychology, vol. 17, pp. 313-326, 1992.

[33] J. B. Moore and R. B. Mosher, "Adjustment responses of children and their mothers to cancer: Self-care and anxiety.," Oncology Nursing Forum, vol. 24, pp. 519-536, 1997.

[34] J. W. Varni, O. J. Sahler, E. R. Katz, R. Mulhern, D. R. Copeland, R. B. Noll, S. Phipps, M. J. Dolgin, and K. Roghmann, "Maternal problem-solving therapy in pediatric cancer," Journal of Psychosocial Oncology, vol. 16, pp. 41-71, 1999.

[35] R. P. Hastings, "Parental stress and behaviour problems of children with developmental disability," Journal of Intellectual Disability, vol. 27, pp. 149-160, 2002.

[36] L. C. Ong, V. Chandran, and N. Y. Boo, "Comparison of parenting stress between Malaysian mothers of four-year-old very low birthweight and normal birthweight children," Acta Paediatrica, vol. 90, pp. 1464-1469, 2001.

[37] E. L. Essex, M. M. Seltzer, and M. Krauss, "Differences in coping effectiveness and well-being among aging mothers and fathers of adults with mental retardation," American Journal of Mental Retardation, vol. 104, pp. 545-563, 1999.

[38] A. E. Kazak, A. Prusak, M. McSherry, S. Simms, D. Beele, M. Rourke, M. Alderfer, and B. Lange, "The Psychosocial Assessment Tool (PAT)(C: Pilot data on a brief screening instrument for identifying high risk families in pediatric oncology.," Families, Systems, \& Health, vol. 19, pp. 303-317, 2001

[39] S. H. Saw, The population of Malaysia. Singapore: Institute of Southeast Asian Studies, 2007. 